\title{
A Stable Magnesium Bromosilylenoid : Transmetalation of a Lithium Bromosilylenoid by Magnesium Bromide
}

Young Mook Lim, Hyeon Mo Cho, and Myong Euy Lee*, Kyoung Koo Baeck ${ }^{\dagger}$

Department of Chemistry, Yonsei University Wonju Campus, Gangwon-do 220-710, Korea

${ }^{\dagger}$ Department of Chemistry, Kangnung National University, Gangnung, Gangwon-do, 210-702, Korea 
${ }^{29} \mathrm{Si}$ NMR of Compound 2 at $-70{ }^{\circ} \mathrm{C}$

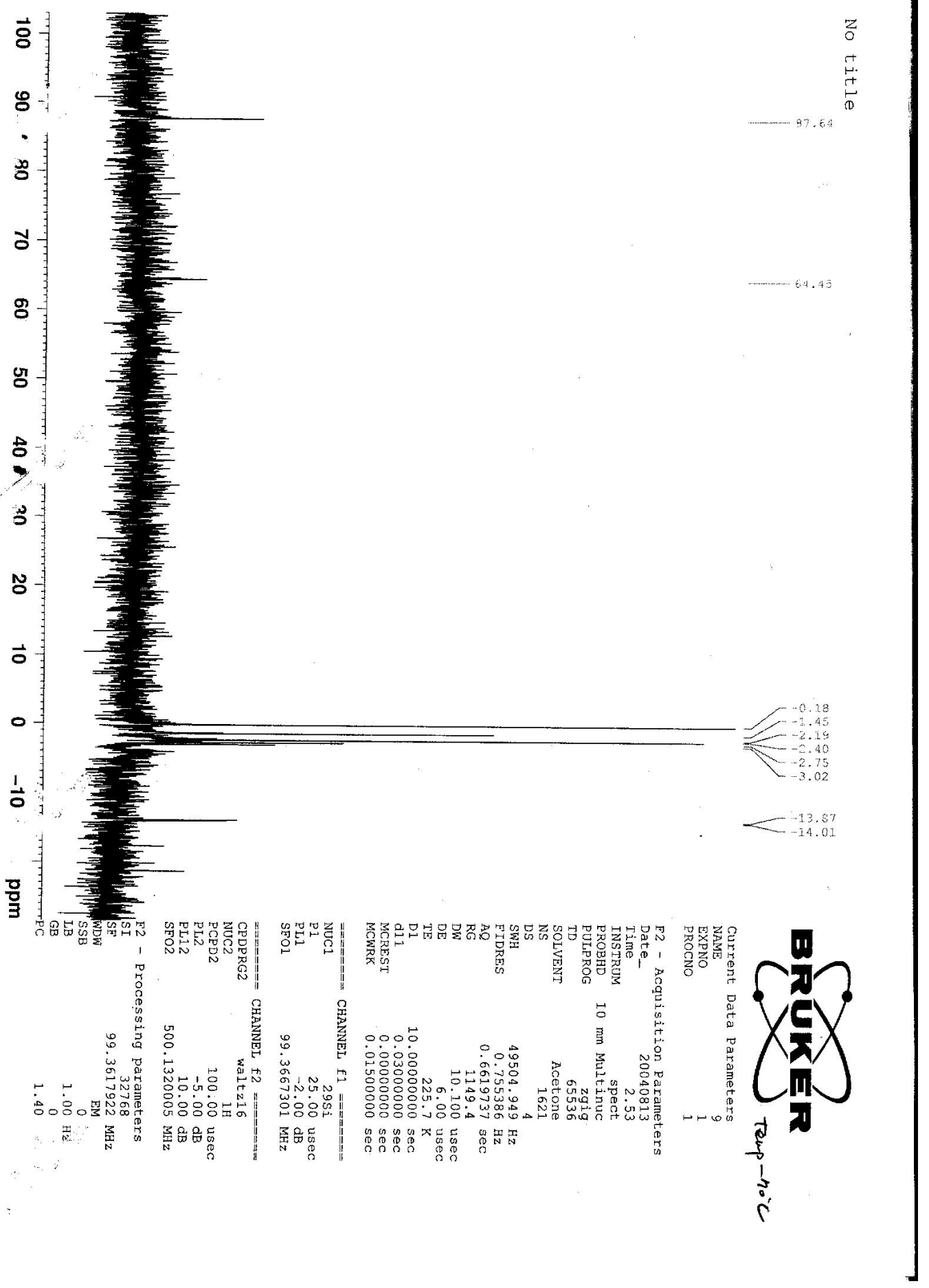


${ }^{29} \mathrm{Si}$ NMR of Compound 3 at $-70{ }^{\circ} \mathrm{C}$
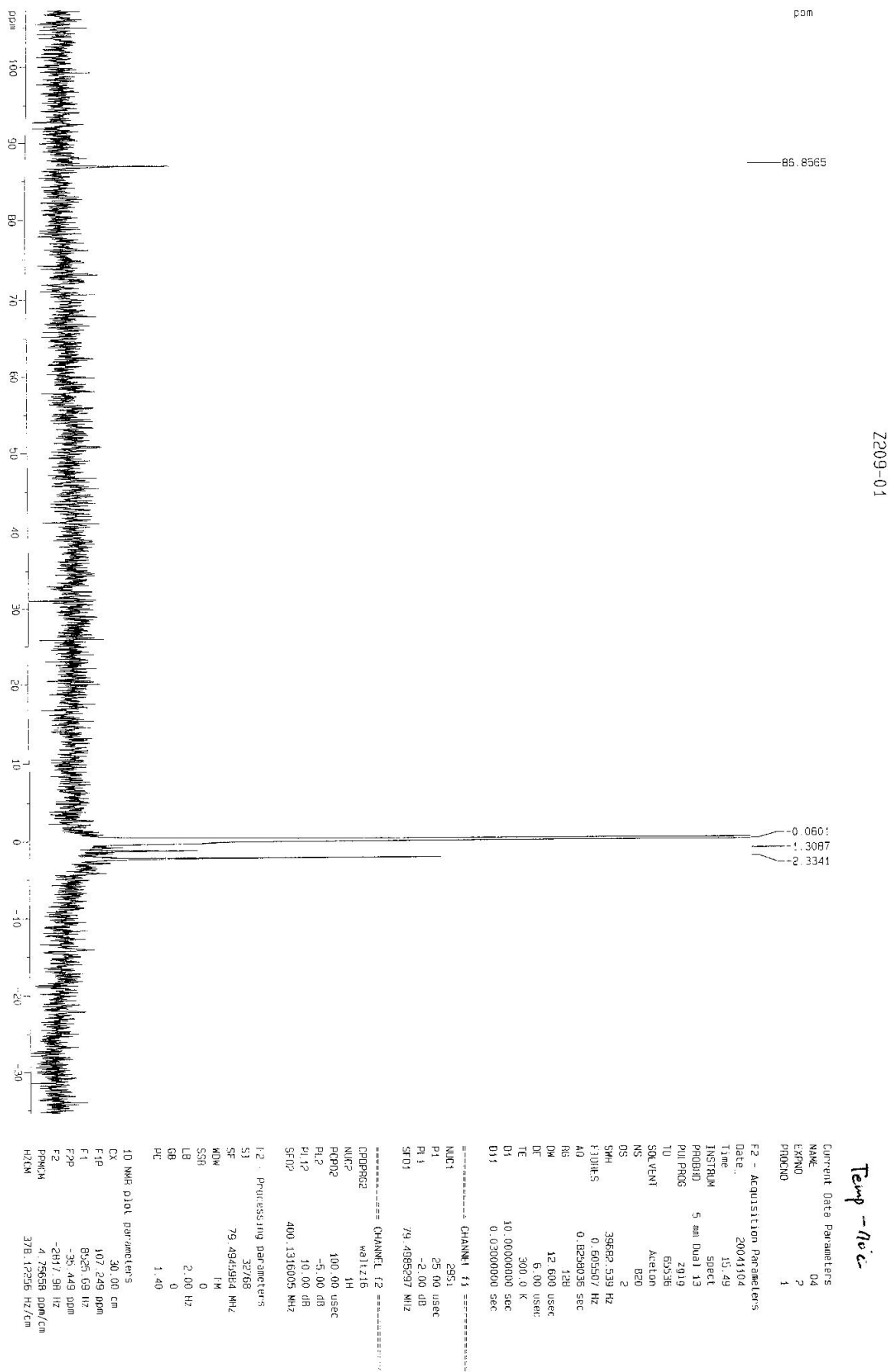
${ }^{29} \mathrm{Si}$ NMR of Compound 3 at $0{ }^{\circ} \mathrm{C}$

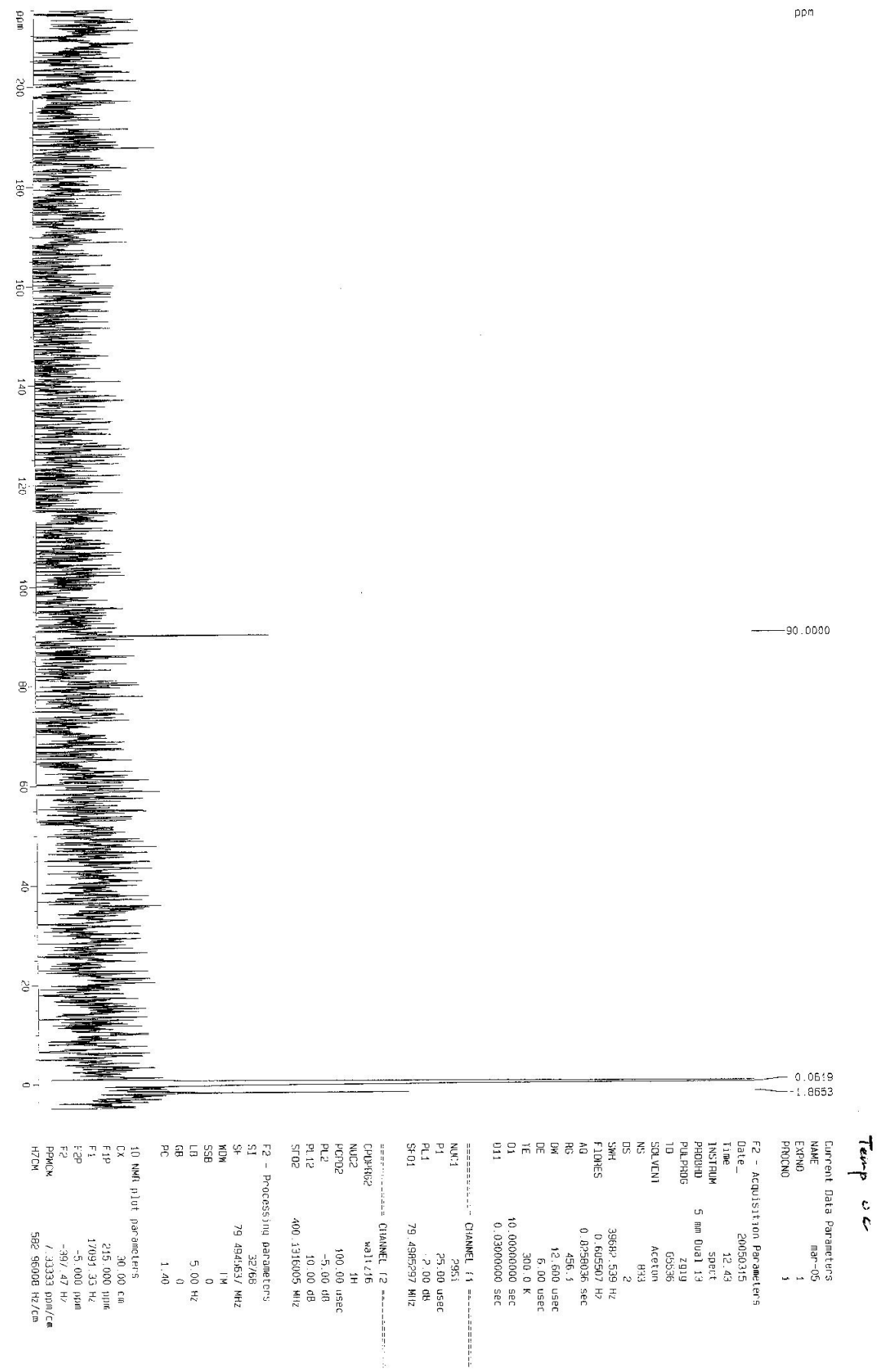


${ }^{29} \mathrm{Si}$ NMR of Compound 4 at $0{ }^{\circ} \mathrm{C}$
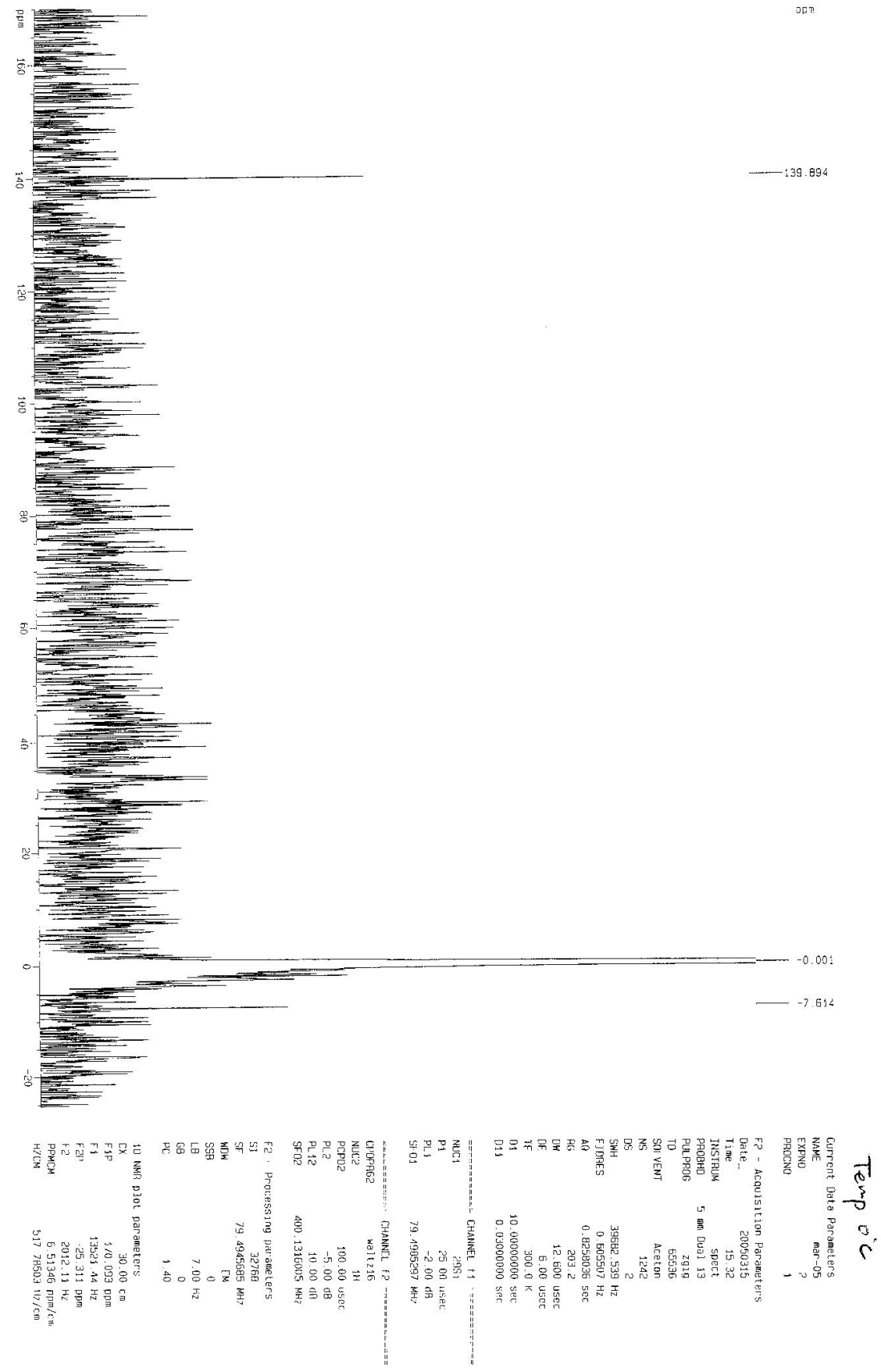
${ }^{29} \mathrm{Si}$ NMR of Compound $\mathbf{1 2}$ at $-70{ }^{\circ} \mathrm{C}$

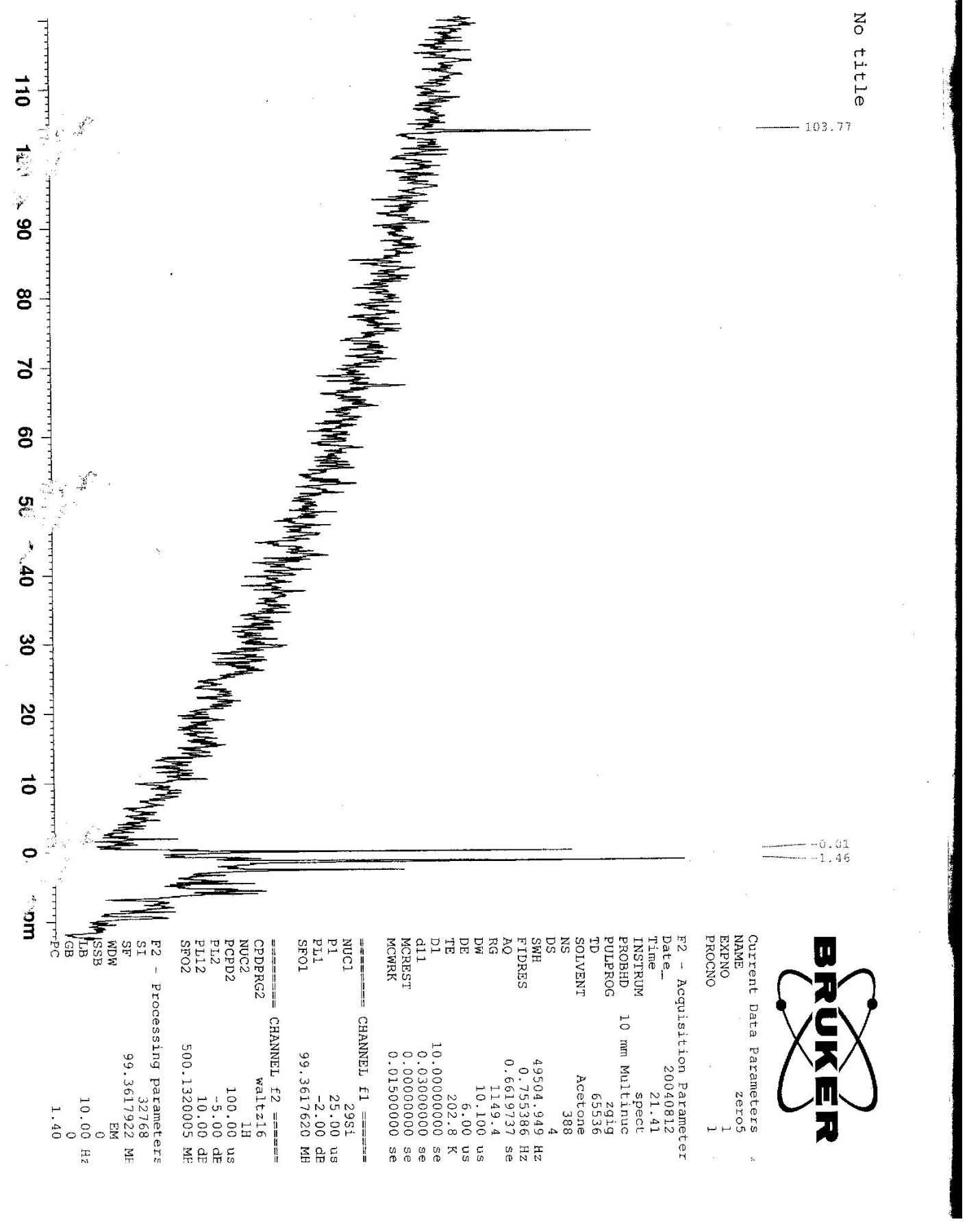




\section{Calculations of the structures 2', 3' and 4'}

All of the computational results presented in the main text are obtained by using the Gaussian 03 suit of programs. The geometry of each structure is optimized by the B3LYP/6-31+G(d) method, and the NMR chemical shift of ${ }^{29} \mathrm{Si}$ is calculated by the GIAO-B3LYP/6-31+G(d) method at the optimized geometry. The input geometry and some of main computational results are collected below, as the form of archived file automatically generated by the Gaussian 03 program.

2': $\delta^{29} \mathrm{Si}=84 \mathrm{ppm}$.

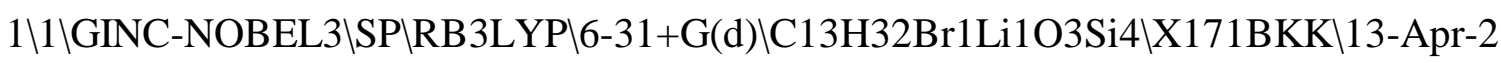

006\0\\# B3LYP/6-31+G(D) NMR TEST\\*** (h3si)3-c-si-br_Ph-Li-3(DME) **

$* * \||0,1 \backslash \mathrm{C}, 0,1.735291,-1.639084,0.716094| \mathrm{Si}, 0,0.50651,-0.211932,0.12992$

8\Si,0,1.192968,-3.324115,0.053265\Si,0,1.585737,-1.725422,2.591924\Si

,0,3.540911,-1.375321,0.220333ไH,0,1.649497,-4.407619,0.975311\H,0,1.7

$64763,-3.620184,-1.284551 \mathrm{H}, 0,-0.289548,-3.426007,-0.026765 \backslash \mathrm{H}, 0,2.6579$

$62,-2.565914,3.196411 \backslash \mathrm{H}, 0,0.275297,-2.31234,3.004511 \backslash \mathrm{H}, 0,1.671661,-0.3$

81546,3.230462\H,0,4.329688,-2.624965,0.444855\H,0,4.181598,-0.310369,

$1.038435 \backslash \mathrm{H}, 0,3.664824,-1.02786,-1.217233 \backslash \mathrm{C}, 0,1.613981,1.377744,0.04455$

6\C,0,2.277396,1.870986,-1.095454\C,0,1.715859,2.148361,1.221997\C,0,3 
$.024662,3.052773,-1.055787 \backslash \mathrm{H}, 0,2.21794,1.315883,-2.028077 \backslash \mathrm{C}, 0,2.465079$

,3.327666,1.273839\H,0,1.205981,1.818234,2.126791\C,0,3.123454,3.7872,

$0.129375 \backslash \mathrm{H}, 0,3.531965,3.39959,-1.954038 \backslash \mathrm{H}, 0,2.53297,3.887554,2.204654 \backslash$

H,0,3.706225,4.704972,0.16144\Br,0,0.318646,-0.733808,-2.226516LLi,0,-

$2.085251,0.244382,0.291997 \backslash \mathrm{C}, 0,-4.490521,-1.52104,0.821766 \backslash \mathrm{H}, 0,-5.3755$

$18,-1.660801,0.184706 \backslash H, 0,-4.351815,-2.414896,1.445844 \backslash \mathrm{H}, 0,-4.62926,-0$

$.646127,1.45917 \backslash \mathrm{C}, 0,-3.036804,-2.37214,-0.856862 \backslash \mathrm{H}, 0,-3.877776,-2.5395$

74,-1.544609\H,0,-2.141552,-2.103014,-1.418441\Н,0,-2.845863,-3.285391

,-0.279679\C,0,-1.530624,2.591809,-1.469744\H,0,-1.050024,2.864899,-0.

$529222 \backslash \mathrm{H}, 0,-0.774126,2.208463,-2.162699 \backslash \mathrm{H}, 0,-2.031308,3.469861,-1.901 \backslash$

$\mathrm{C}, 0,-3.186184,1.139778,-2.350144 \backslash \mathrm{H}, 0,-3.909346,0.38502,-2.035921 \backslash \mathrm{H}, 0,-$

3.712417,1.98437,-2.816537\H,0,-2.479717,0.700892,-3.06546\C,0,-3.3233

23,2.475972,1.935518\H,0,-3.679038,2.664454,0.921489\Н,0,-4.162193,2.5

$31509,2.643448 \backslash \mathrm{H}, 0,-2.569613,3.227314,2.208813 \backslash \mathrm{C}, 0,-2.226188,0.799739$,

$3.217525 \backslash \mathrm{H}, 0,-1.791579,-0.195542,3.113495 \backslash \mathrm{H}, 0,-1.444115,1.504077,3.530$

053\H,0,-3.02789,0.784089,3.969002\O,0,-2.499149,1.576019,-1.175677\O,

0,-3.333974,-1.282825,0.025141\O,0,-2.752219,1.169002,1.941166\\Versio

$\mathrm{n}=\mathrm{IBM} 64-\mathrm{G} 03 \mathrm{RevC} .02 \backslash \mathrm{State}=1-\mathrm{A} \backslash \mathrm{HF}=-4477.6610118 \backslash \mathrm{RMSD}=4.148 \mathrm{e}-09 \backslash \mathrm{Dipole}=-3$ 
3': $\delta^{29} \mathrm{Si}=92 \mathrm{ppm}$.

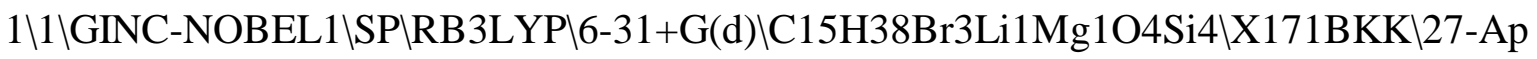

r-2006\0\\# B3LYP/6-31+G(D) NMR \* (h3si)3-c-si-brPh-Li3(Me2O)-MeBr2(

DME)*\\0,1\C,0,1.936877,1.745358,-1.782897\Si,0,1.005759,0.870181,-0.3

14172\Si,0,1.427009,1.029197,-3.467858\Si,0,3.771053,1.338848,-1.54930

$5 \backslash \mathrm{Si}, 0,1.778567,3.628383,-1.894399 \backslash \mathrm{H}, 0,2.540669,1.220038,-4.442023 \backslash \mathrm{H}, 0$

,0.240351,1.74228,-4.011613\H,0,1.131066,-0.420781,-3.376712〈H,0,4.621

$59,2.243334,-2.370875 \backslash H, 0,4.070011,-0.060197,-1.960293 \backslash H, 0,4.195242,1$.

490519,-0.130009\Н,0,2.291437,4.09877,-3.213916\Н,0,2.576125,4.312296,

$-0.84188 \backslash \mathrm{H}, 0,0.366427,4.077867,-1.789743 \backslash \mathrm{C}, 0,1.049558,2.073804,1.17711$

7\C,0,0.331954,3.278938,1.304268\C,0,1.889699,1.710842,2.246169\C,0,0.

493158,4.110226,2.415454\H,0,-0.347468,3.587455,0.51408\C,0,2.048778,2

$.529499,3.36843 \backslash \mathrm{H}, 0,2.41638,0.760106,2.213469 \backslash \mathrm{C}, 0,1.346369,3.732955,3$.

457935\Н,0,-0.060241,5.045164,2.476709\H,0,2.705038,2.21698,4.177477\Н 
,0,1.464185,4.373329,4.328996\Br,0,-1.297154,1.206154,-1.006368\Li,0,-

$3.204468,-0.153135,0.021634 \backslash \mathrm{C}, 0,-5.298608,-1.982473,-1.01403 \backslash \mathrm{H}, 0,-5.63$

$4538,-2.039371,-2.05868 \backslash \mathrm{H}, 0,-5.501843,-2.942434,-0.519722 \backslash \mathrm{H}, 0,-5.83430$

2,-1.180739,-0.501565\С,0,-3.093745,-2.648665,-1.629766\Н,0,-3.347466,

$-2.669423,-2.697496 \backslash \mathrm{H}, 0,-2.048034,-2.367569,-1.505312 \backslash \mathrm{H}, 0,-3.254311,-3$

$.641931,-1.192379 \backslash \mathrm{C}, 0,-4.751206,2.3166,0.563734 \backslash \mathrm{H}, 0,-4.663851,1.873486$

$, 1.557235 \backslash \mathrm{H}, 0,-3.976326,3.082585,0.428477 \backslash \mathrm{H}, 0,-5.744664,2.770945,0.450$

495\C,0,-4.73844,1.704535,-1.731814\Н,0,-4.498334,0.860341,-2.380378\Н

,0,-5.775214,2.024868,-1.901889\H,0,-4.053909,2.533537,-1.950367\C,0,-

$2.243226,0.492985,2.749412 \backslash \mathrm{H}, 0,-2.015476,1.376718,2.151601 \backslash \mathrm{H}, 0,-2.8517$

$54,0.770494,3.621379 \backslash \mathrm{H}, 0,-1.30901,0.022639,3.070804 \backslash \mathrm{C}, 0,-3.232964,-1.6$

$67275,2.550469 \backslash \mathrm{H}, 0,-3.775486,-2.290801,1.836481 \backslash \mathrm{H}, 0,-2.28533,-2.146884$

,2.817358\H,0,-3.847577,-1.514568,3.448402\O,0,-4.577687,1.260032,-0.3

81475\O,0,-3.907891,-1.674593,-0.954542\O,0,-2.982819,-0.409705,1.9089

$75 \backslash \mathrm{Mg}, 0,1.318997,-1.734551,0.276222 \backslash \mathrm{Br}, 0,0.631921,-2.028181,2.630962 \backslash \mathrm{B}$

r,0,0.611994,-3.249959,-1.518778\O,0,3.410029,-2.11416,0.369893\C,0,4.

1973,-1.844977,1.538642\H,0,5.034954,-1.186933,1.276869\H,0,3.546651,-

$1.363624,2.268847 \backslash \mathrm{H}, 0,4.57515,-2.785261,1.957952 \backslash \mathrm{C}, 0,4.142431,-2.83131$ 
,-0.638418\H,0,4.982132,-2.220734,-0.990062\H,0,4.511931,-3.775681,-0.

22035\H,0,3.450499,-3.034658,-1.455277\|Version=IBM64-G03RevC.02\State

$=1-\mathrm{A} \backslash \mathrm{HF}=-9976.486346 \mathrm{RMSD}=7.225 \mathrm{e}-09$ \ipole $=-2.9380043,0.8825775,0.5596$

475\PG=C01 [X(C15H38Br3Li1Mg1O4Si4)]\@

$4^{\prime}: \delta^{29} \mathrm{Si}=139 \mathrm{ppm}$.

1\1\GINC-NOBEL6ISPIRB3LYP16-31+G(d)\C9H20Br2Mg1O1Si4IX171BKK105-Apr-20

0610\॥ B3LYP/6-31+G(D) NMR TESTII**** (h3si)3-c-si-(Ph)-MgBr2-DME ***

$* \mid \backslash 0,1 \backslash \mathrm{C}, 0,-1.94882,-1.527372,-0.585562 \backslash \mathrm{Si}, 0,-0.719678,-0.063574,-0.36$

2566\Si,0,-0.95475,-3.143737,-0.629903\Si,0,-2.772426,-1.353712,-2.295

809\Si,0,-3.304616,-1.751272,0.736671\H,0,-1.770967,-4.250356,-1.19631

4\H,0,-0.52084,-3.549687,0.732654\Н,0,0.267407,-3.001422,-1.471631\H,0

,-3.679897,-2.507818,-2.533088\H,0,-1.738231,-1.348283,-3.366727\H,0,-

$3.563772,-0.10315,-2.397284 \backslash \mathrm{H}, 0,-3.522839,-3.205884,0.975259 \backslash \mathrm{H}, 0,-4.60$

2534,-1.187057,0.27504\H,0,-2.941185,-1.115479,2.02481\C,0,-1.61939,1.

$583863,-0.098943 \backslash \mathrm{C}, 0,-3.006043,1.772544,0.053737 \backslash \mathrm{C}, 0,-0.807112,2.73827$ 
8,-0.129645\C,0,-3.558241,3.048117,0.190598\Н,0,-3.679701,0.921672,0.0

63363\C,0,-1.351999,4.016227,0.007464\H,0,0.26758,2.648925,-0.278835\C

$, 0,-2.730257,4.173966,0.175058 \backslash \mathrm{H}, 0,-4.633184,3.161534,0.308964 \backslash \mathrm{H}, 0,-0$.

700407,4.885953,-0.01999\H,0,-3.15816,5.167928,0.281093\Mg,0,1.846045,

$0.025549,-0.040293 \backslash \mathrm{Br}, 0,3.253425,1.868221,-0.689545 \backslash \mathrm{Br}, 0,0.217706,-0.3$

89973,2.016137\O,0,3.069141,-1.639254,-0.016296\C,0,4.279743,-1.683442

,-0.803263ไH,0,4.158521,-2.401782,-1.621858\H,0,4.443743,-0.677423,-1.

$19028 \backslash \mathrm{H}, 0,5.116518,-1.976887,-0.16026 \backslash \mathrm{C}, 0,2.804851,-2.873354,0.677685 \backslash$

H,0,2.641646,-3.677375,-0.048076\H,0,3.655111,-3.11388,1.325558\H,0,1 .

910114,-2.721579,1.280495।|Version=IBM64-G03RevC.02।State=1-AlHF=-6931

$.9179228 \backslash$ RMSD $=6.056 \mathrm{e}-09$ \ipole $=0.3982453,-1.9380595,-0.1558159 \backslash \mathrm{PG}=\mathrm{C} 01$

[X(C9H20Br2Mg1O1Si4)]॥@

Full reference 19b : Gaussian 03, Revision B.04, Frisch, M. J.; Trucks, G. W.; Schlegel, H. B.;

Scuseria, G. E.; Robb, M. A.; Cheeseman, J. R.; Montgomery, Jr., J. A.; Vreven, T.; Kudin, K. N.;

Burant, J. C.; Millam, J. M.; Iyengar, S. S.; Tomasi, J.; Barone, V.; Mennucci, B.; Cossi, M.; Scalmani, G.; Rega, N.; Petersson, G. A.; Nakatsuji, H.; Hada, M.; Ehara, M.; Toyota, K.; Fukuda, R.; Hasegawa, J.; Ishida, M.; Nakajima,; Honda, T. Y.; Kitao, O.; Nakai, H.; Klene, M.; Li, X.; Knox, J. E.; Hratchian, H. P.; Cross, J. B.; Adamo, C.; Jaramillo, J.; Gomperts, R.; Stratmann, R. E.; Yazyev, O.; Austin, A. J.; Cammi, R.; Pomelli, C.; Ochterski, J. W. ; Ayala, P. Y.; Morokuma, K.; Voth, G. A.; Salvador, P.; Dannenberg, J. J.; Zakrzewski, V. G. ; Dapprich, S.; Daniels, A. D.; Strain, M. C.; Farkas, O.; Malick, 
D. K.; Rabuck, A. D.; Raghavachari, K.; Foresman, J. B.; Ortiz, J. V.; Cui, Q.; Baboul, A. G.; Clifford, S.; Cioslowski, J.; Stefanov, B. B.; Liu, G.; Liashenko, A.; Piskorz, P.; Komaromi, I.; Martin, R. L.; Fox, D. J.; Keith, T.; Al-Laham, M. A.; Peng, C. Y.; Nanayakkara, A.; Challacombe, M.; Gill, P. M. W.; Johnson, B.; Chen, W.; Wong, M. W.; Gonzalez, C.; Pople, J. A. Gaussian, Inc., Pittsburgh PA, 2003. 